\title{
Observed Effects of Sheltered Instruction Observation Protocol (SIOP) Methods in Education of Students in Uganda
}

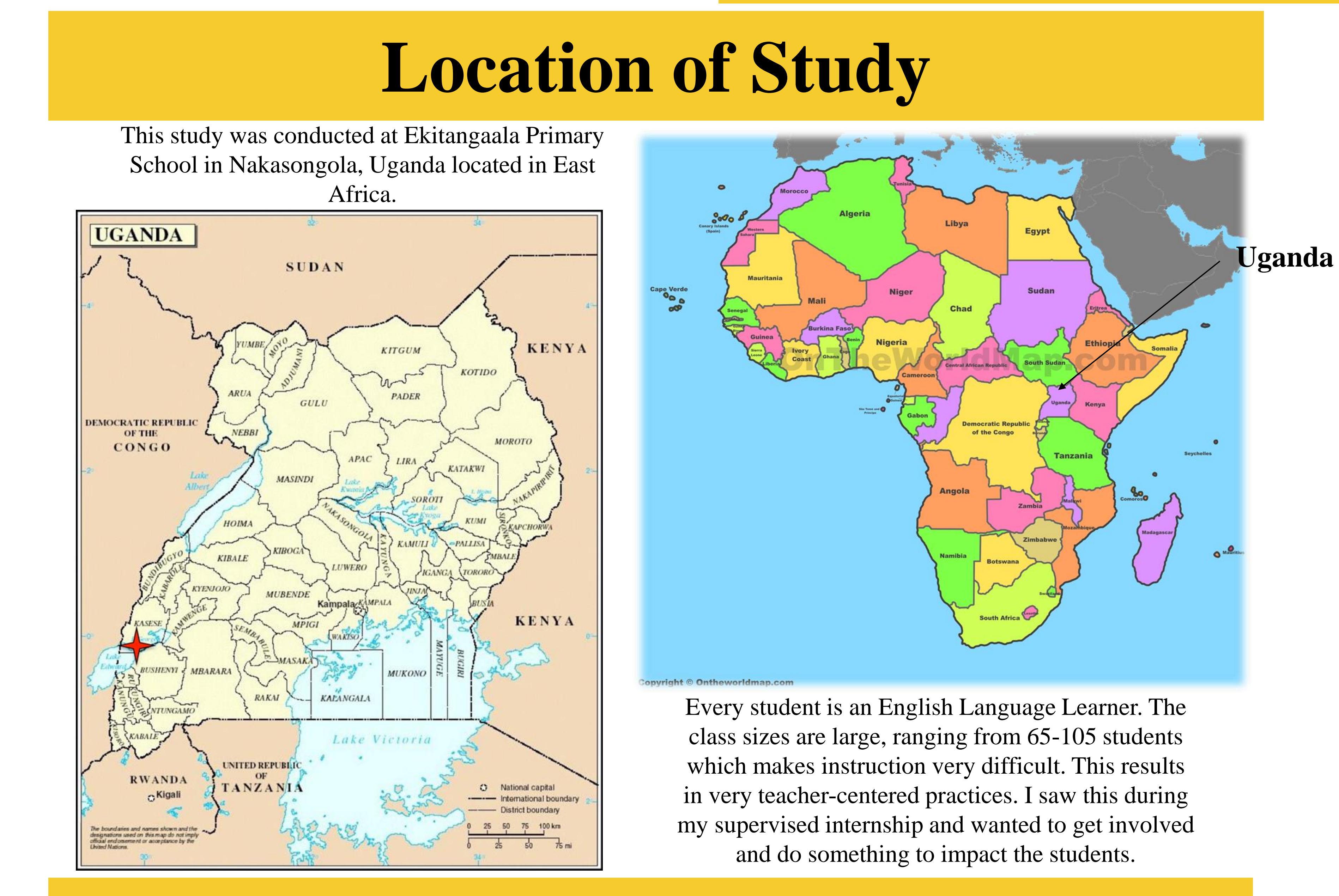

Abstract of the Study

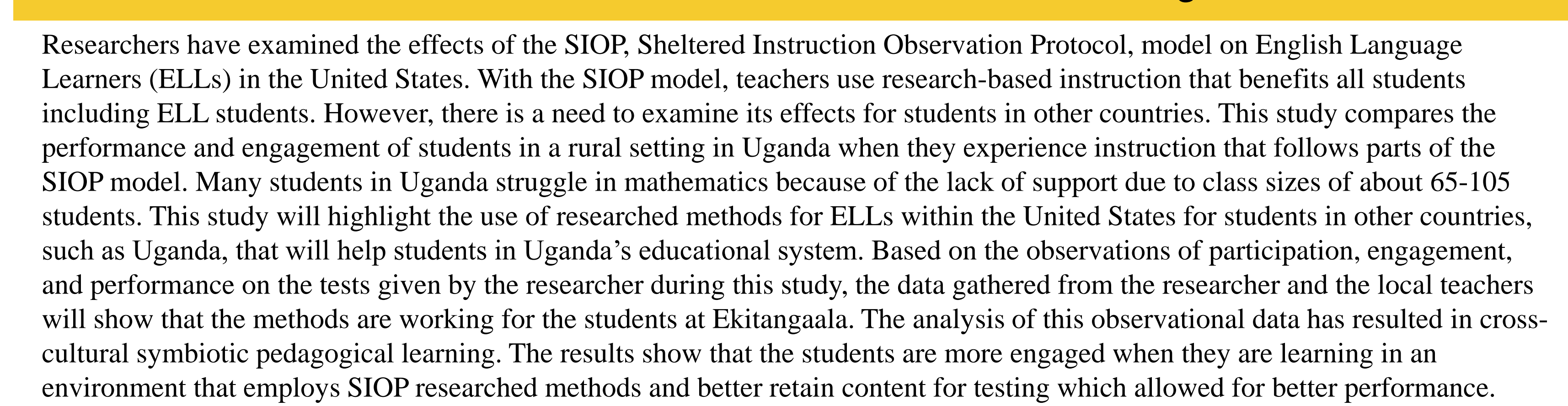

Typical classroom in Ekitangaala Primary School
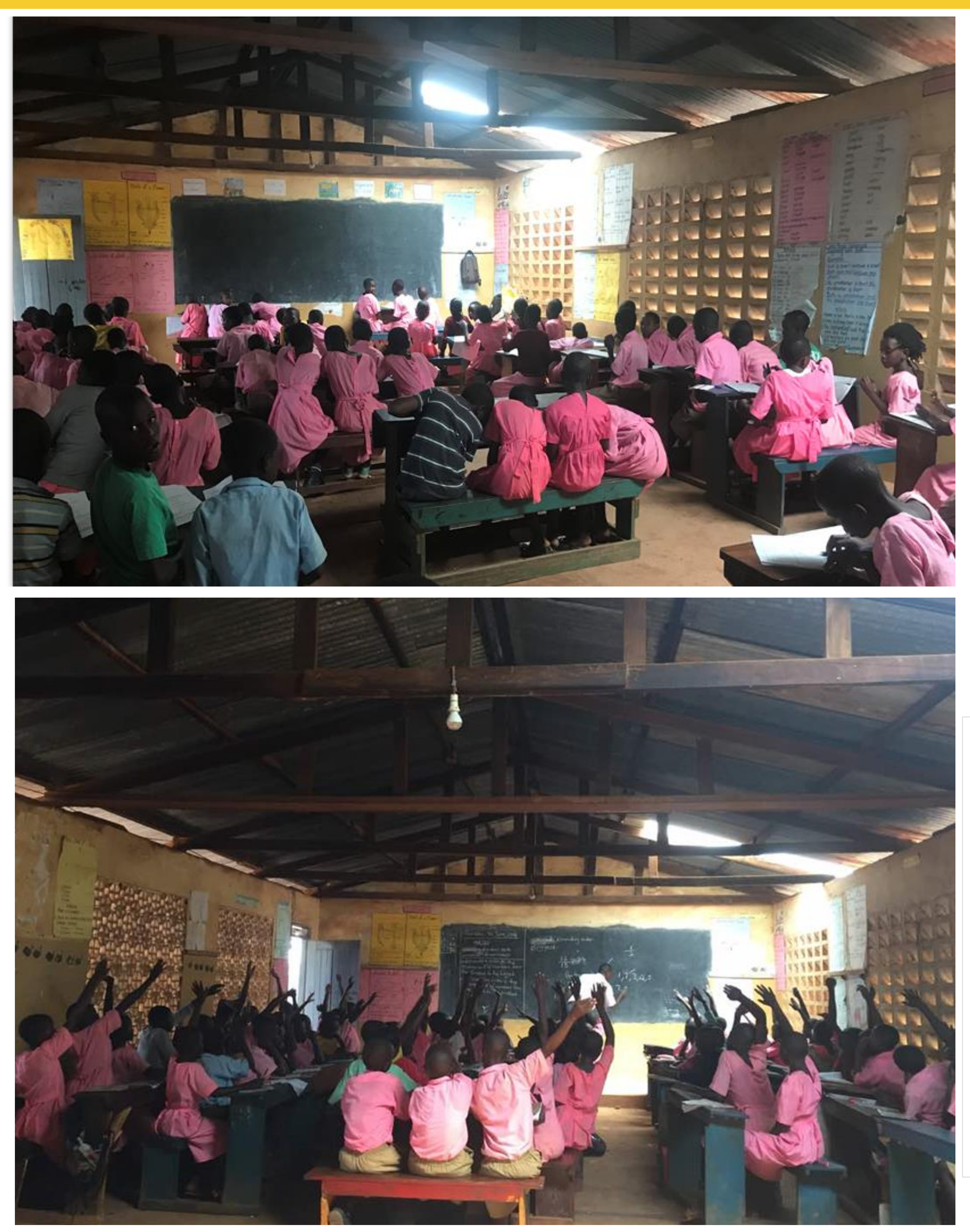

Ms. Amanda Whitlock and Dr. Ikechukwu Ukeje Bagwell College of Education

Kennesaw State University, Kennesaw Georgia.

Figure 1. Base-line Student Performance in Subjects - Pie Charts

Primary 5 Math Baseline Primary 6 Science Baseline
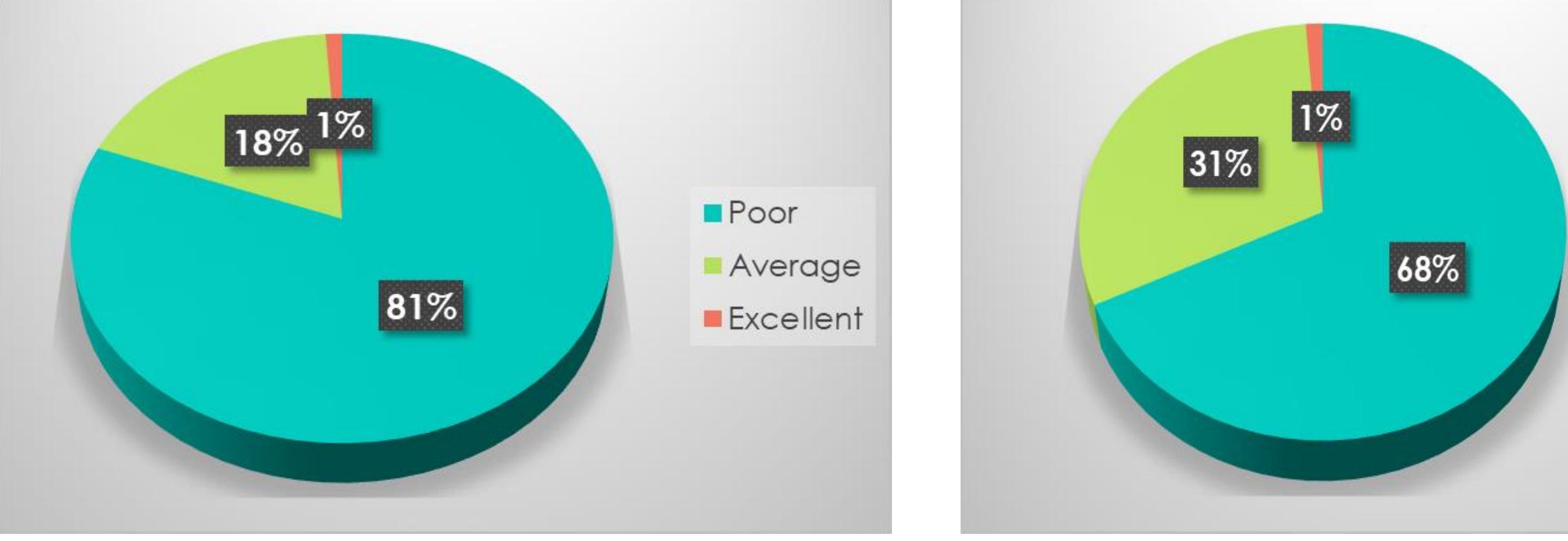

Primary 7 English Baseline

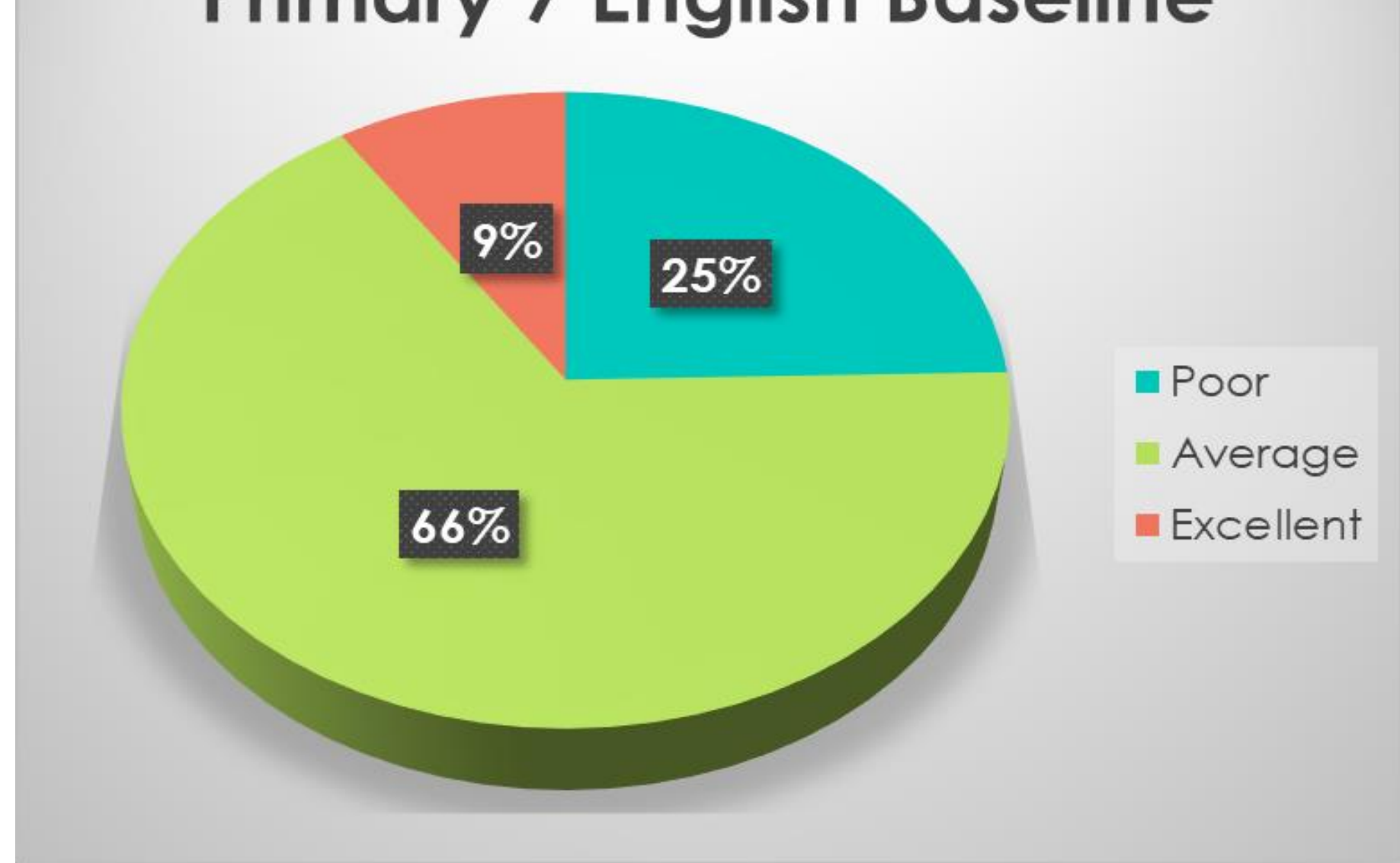

Figure 2. After Intervention Student Performance in Subjects - Pie Charts
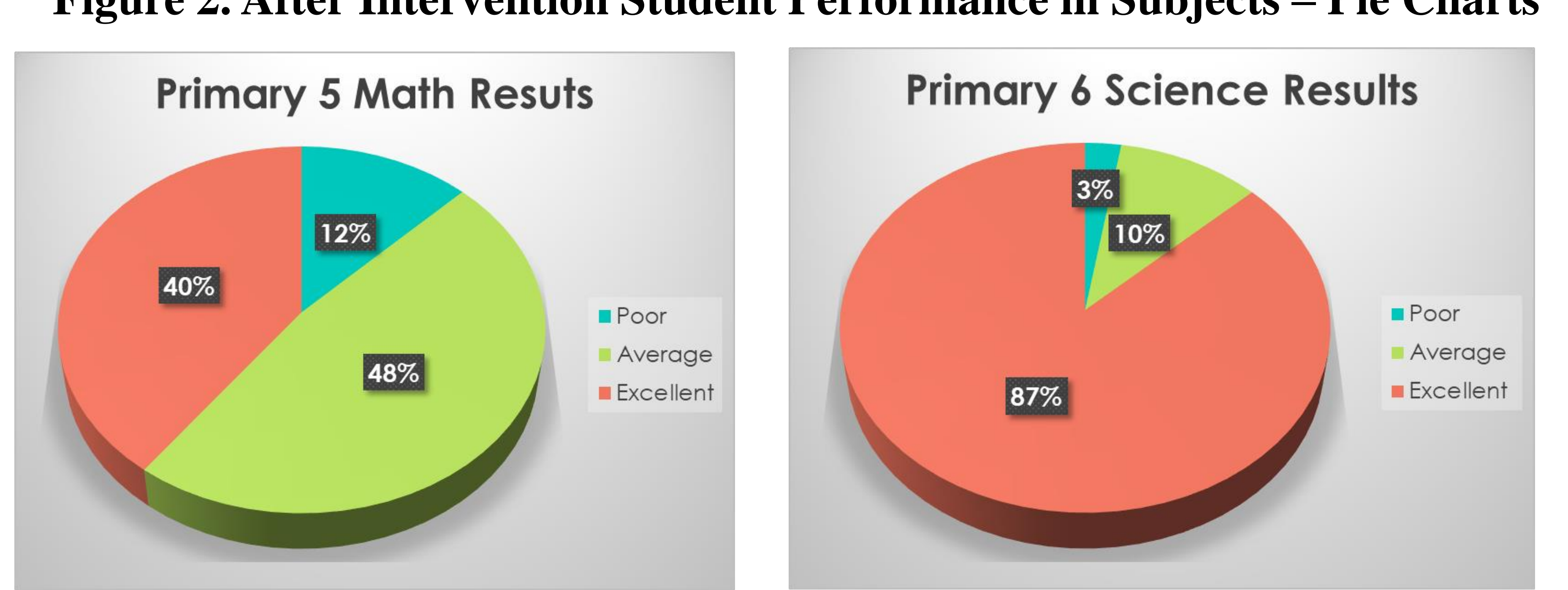

Primary 7 English Results

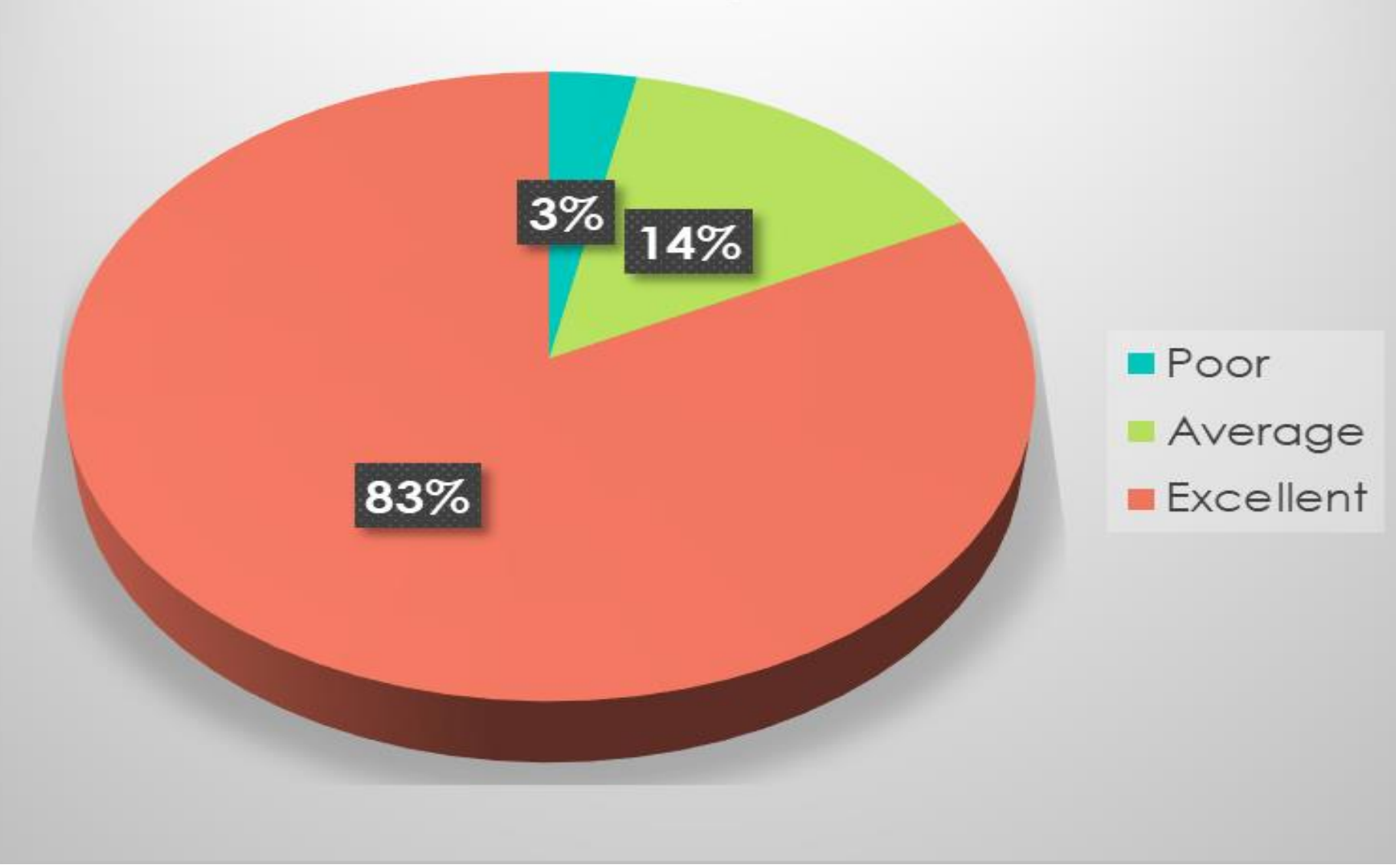

Figure 3. Student Performance in All Three Subjects: Baseline and After Intervention Testing Student Performance in All Three Subjects: Baseline Testing Student Performance Across All Three Sujjects

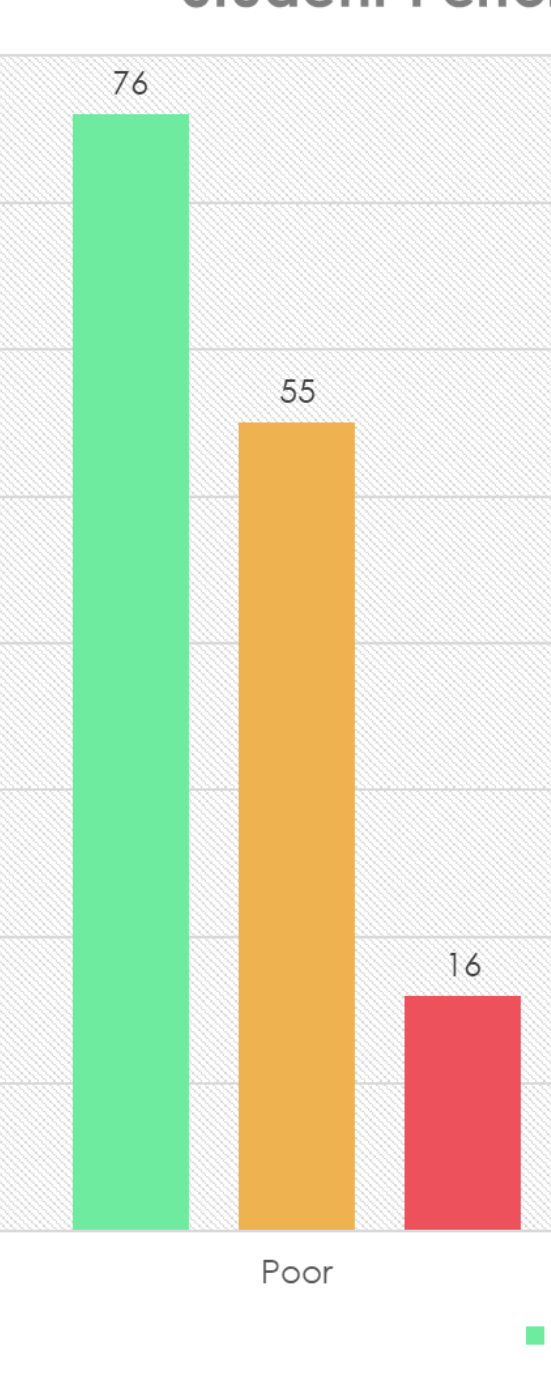

Intervention Classes

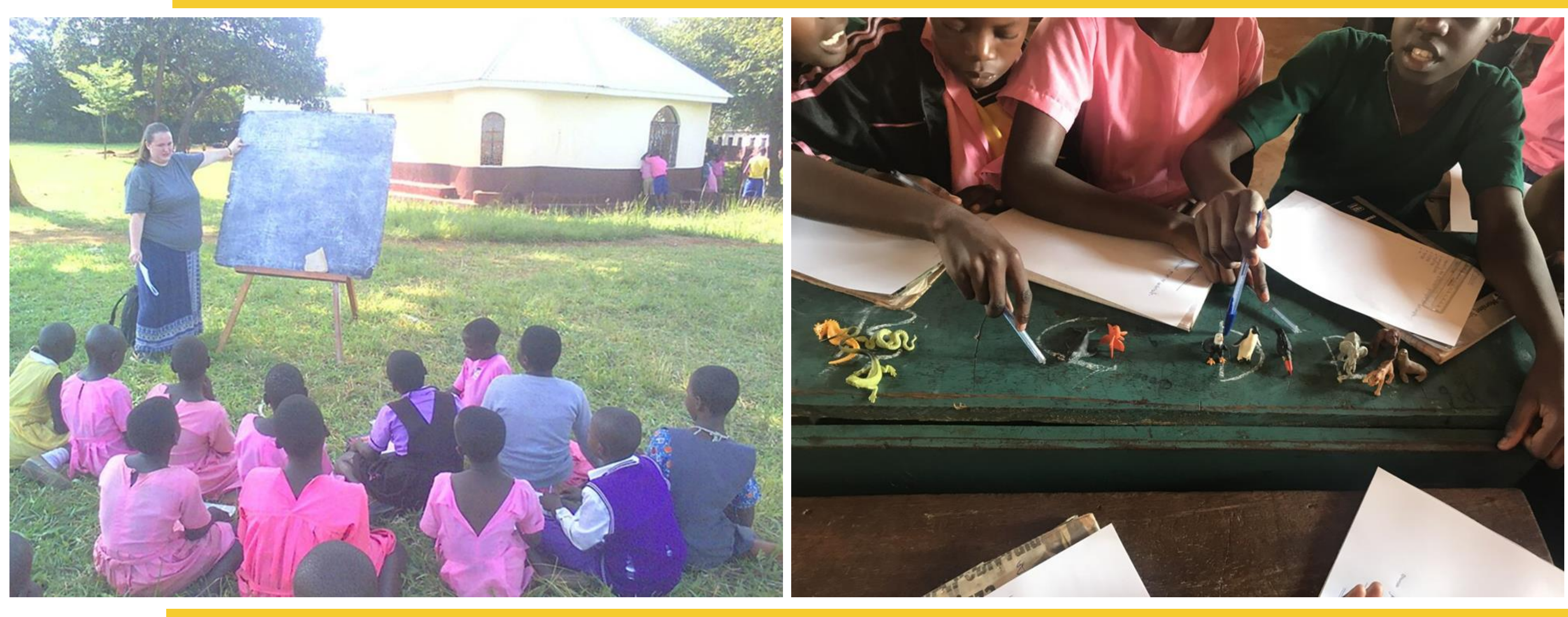

Administration and Teacher Support

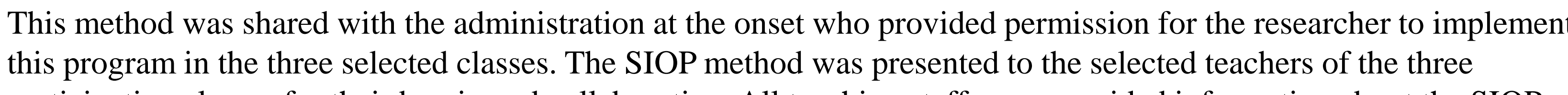
participating classes for their buy in and collaboration. All teaching staff were provided information about the SIOP
method during a staff development workshop day. KSU Professor, Dr. Ikechukwu UKkeje approved all aspects of this

Sheltered Instruction Observation Protocol (SIOP)



